\title{
HACIA UN MARCO ANALÍTICO Y METODOLÓGICO PARA LA EVALUACIÓN DEL MEJORAMIENTO EDUCATIVO EN ESCUELAS CHILENAS $^{1}$
}

\author{
Luis Felipe De la Vega
}

RESUMEN

En este trabajo se presentan los resultados de una investigación que tuvo como objetivo el desarrollo de una aproximación metodológica que permitiera evaluar la presencia de atributos de mejoramiento educativo en las escuelas chilenas. Para ello, se aplicó la metodología de análisis multicriterio Método Analítico Jerárquico (Analytical Hierarchical Process, AHP), una herramienta que busca identificar criterios para la evaluación de un determinado fenómeno, a partir de la identificación y discusión de expertos. En este caso, los expertos consultados fueron docentes, directores de establecimientos, asesores técnico-educativo y académicos, quienes discutieron y analizaron los elementos que caracterizarían a una escuela chilena que está desarrollando procesos de mejoramiento. Como resultado de la investigación se pudo elaborar una matriz jerárquica de criterios de evaluación, validada por los expertos, y una propuesta de indicadores y recursos de información que permitirían implementar el proceso evaluativo. Finalmente, se realizó un análisis de los resultados obtenidos en la investigación y se identificaron debilidades y proyecciones para su implementación en diferentes contextos socioeducativos.

Palabras clave: mejoramiento educativo, evaluación educativa, investigación evaluativa, calidad de la educación.

\section{TOWARDS AN ANALYTICAL AND METHODOLOGICAL FRAMEWORK FOR THE SCHOOL IMPROVEMENT ASSESSMENT IN CHILEAN SCHOOLS}

\section{ABSTRACT}

This paper presents the results of a study that aimed to develop a methodological approach to evaluate improvement process results in Chilean Schools. To this objective, the multicriteria analysis methodology Analytical Hierarchical Process AHP was applied. This is a tool that identifies criteria for the evaluation of a phenomenon considering the identification and the discussion of experts. In this case, the experts consulted were teachers, school principals, technical advisors and scholars, who discussed and analyzed the characteristics of school improvement in Chilean Schools. As a result of the investigation was possible to develop a hierarchical matrix with evaluation criteria, validated by experts, and propose indicators and information resources that allowed to implementing the evaluation process. Finally, an analysis of the results of the research, weakness and projections for this methodology implementation in different contexts was made.

Keywords: educational improvement, educational assessment, evaluation research, quality of education.

1 Este estudio ha sido financiado por la Convocatoria de apoyo a la investigación en educación 2013 del CNED.

2 Facultad de Educación, Pontificia Universidad Católica de Chile, Santiago, Chile. Contacto: ldelaveg@uc.cl 


\section{La evaluación de las escuelas y el mejoramiento educativo}

De la intensidad de la discusión acerca de la educación pública en Chile es clara la necesidad de contar con un sistema educativo capaz de combinar de manera exitosa las necesidades de calidad y equidad (Bellei, Valenzuela y De los Ríos, 2010; Martinic, 2010). El Estado chileno ha generado progresivamente sistemas y mecanismos con los que espera avanzar hacia ese objetivo. Uno de los desafíos que ha tenido mayor relevancia a nivel de sistema educativo es la incorporación de la evaluación, entendida como mecanismo que favorece el mejoramiento de la acción y los resultados.

Durante los últimos 15 años ha sido posible observar en Chile la profundización en la centralidad del desempeño de las instituciones y actores educativos como objeto fundamental de análisis, y la existencia de incentivos asociados al desempeño, en tanto mecanismo movilizador del mejoramiento (Weinstein \& Muñoz, 2012). Este camino se corona con la Ley 20.529, que define un Sistema Nacional de Aseguramiento de la Calidad de la Educación Escolar, recientemente implementado.

Frente a la opción de evaluación considerada por la institucionalidad educativa chilena, y atendiendo el doble desafío de propender a la calidad y equidad de la educación, se vuelve central la discusión acerca de las estrategias que se utilizan para evaluar el cumplimiento de los objetivos educativos de las escuelas, su aporte a las metas del sistema y, sobre todo, al mejoramiento de la escuelas.

Esta investigación tuvo como propósito el diseño de un modelo que permitiera la evaluación del desempeño de las escuelas chilenas, considerando la presencia de atributos de mejoramiento educativo en ellas. A través de este ejercicio se buscó reconocer la complejidad de los procesos de mejoramiento y la relevancia de considerarlos a la hora de analizar el desempeño de una escuela. Es importante mencionar que el presente artículo da cuenta de los resultados de la primera etapa del trabajo investigativo, orientado hacia el diseño de la propuesta metodológica de la evaluación de desempeño. La 
segunda etapa buscará evaluar la propuesta, a partir del estudio de las fortalezas y debilidades metodológicas de su implementación. Por esta razón, el ámbito de análisis relacionado con este último proceso no forma parte del presente reporte.

\section{Mejoramiento educativo: dimensiones relevantes y desafíos para la evaluación de un proceso complejo}

El concepto de mejoramiento educativo ha favorecido el interés en el cambio en educación, entendido como un proceso que involucra a las personas e instituciones que participan de su implementación, preguntándose acerca de qué tiene que ocurrir para modificar favorablemente un escenario educativo, considerando variables externas e internas a la escuela, materiales y simbólicas (Fullan, 1982, 2002; Murillo, 2003; Hopkins, 2009).

Existe consenso respecto de que los procesos de mejoramiento debieran tener como foco la relación entre enseñanza y aprendizaje (Fullan, 2002; Hopkins, 2009; Levin, 2009). Elmore (2010) denomina a esta relación núcleo pedagógico, el cual está compuesto por la interacción dentro del aula entre el docente, el estudiante y los contenidos. Las dinámicas de esa interacción se relacionarían directamente con el nivel de aprendizaje y la calidad educativa. Así, lo central del mejoramiento redunda en lo que ocurre en la sala de clases, lo que releva el rol de los profesionales de la escuela y su vínculo de aprendizaje con los estudiantes.

Junto con lo anterior, Levin (2009) indica que este proceso implica la organización en torno a un propósito moral, es decir, a una meta que tiene una dimensión simbólica y a la vez normativa. Esta idea apunta a que el mejoramiento educativo se puede desarrollar en la medida en que hay finalidades que están más allá de lo meramente técnico y de las obligaciones que las escuelas deben cumplir.

Las transformaciones del funcionamiento de la escuela y la dinámica del núcleo pedagógico favorecen el mejoramiento cuando cuentan, además, con una condición de sustentabilidad o mejoramiento continuo (Hargreaves y Fink, 2006). En esa línea, 
Murillo y Krichesky (2012) sostienen que la literatura acerca del mejoramiento escolar ha ido consolidando la idea de que este ocurre a través de fases no lineales, porque las decisiones presentes en algunos casos ayudan a avanzar, mientras que en otras, provocan retrocesos.

Se puede decir que existe consenso en relación con la estructura central del mejoramiento educativo, recientemente señalada. Junto con ello, sus autores referenciales también tienden a concordar respecto de las debilidades o desafíos para su desarrollo y su logro. Tal vez el mayor de estos sea asumir la complejidad de los escenarios en que se implementa este proceso, manteniendo el foco en el objeto de mejoramiento (Chrispeels \& González, 2006; Bogotch, Mirón \& Biesta, 2007). Por su parte, Hallinger \& Heck (2011) y Datnow \& Park (2004) señalan que las estrategias de mejoramiento operan en escenarios complejos y contextos socioculturalmente diferenciados, por lo que se requiere de análisis multinivel que incluya a los distintos actores e instituciones involucrados y a sus relaciones, las características de la escuela (tamaño, composición, características socioeconómicas), de las políticas o programas cuya implementación se estudia (Anderson, Mascall, Stiegelbauer y Park, 2012).

Teniendo en consideración este fenómeno, Bourke \& McGee (2012) señalan que la lógica de la comprensión del proceso de mejoramiento educativo implica asumir que, pese a que se puede observar que este sigue un patrón o tránsito por etapas, también hay un conjunto amplio de elementos que hacen de este proceso algo complejo e impredecible.

\section{La evaluación del desempeño de las escuelas y los desafíos por vincularla al mejoramiento educativo}

Tal como se ha enunciado, el mejoramiento educativo es un proceso complejo de desarrollar y evaluar. Por esta razón, la evaluación de las escuelas y su desempeño es un área que cuenta con múltiples acercamientos y perspectivas y es objeto de intenso debate (OECD, 2013). 
En relación con su uso para las políticas públicas y la toma de decisiones en particular, se ha observado un tránsito desde el uso de indicadores globales de resultados -tales como los test de aprendizaje estandarizados- hacia la consideración de otros insumos, mecanismos de aproximación al trabajo y desempeño escolar, e indicadores de funcionamiento y resultados de las escuelas (Schwatrz, Hamilton, Stecher \& Steele, 2011; Hargreaves, 2012).

El cambio mencionado ha buscado favorecer reflexiones evaluativas que, por un lado sean más justas y que, por otro, describan con mayor detalle las condiciones y características donde se desarrolle el proceso educativo (Froemel, 2003; Thomas, 2010; Shwartz, Hamilton, Stecher y Steele, 2011). Junto con ello, se han constatado también importantes perjuicios relacionados con la toma de decisiones basadas en uno o pocos indicadores (Dale $\&$ Robertson, 2007), lo que ha conducido a algunos países a cambiar de estrategia de evaluación (Ng, 2012).

De acuerdo con la OECD (2013), la evaluación de una escuela debiera involucrar procesos relevantes tales como enseñanzaaprendizaje, liderazgo, administración, clima y gestión de los recursos humanos. Es decir, se ha ido avanzando hacia la consideración de los procesos de mejoramiento educativo en la evaluación. Esta situación implica la necesidad de asumir el levantamiento de información de carácter procesual. Además, se vuelve relevante considerar que los establecimientos educacionales están en un piso distinto al mejoramiento. Junto con ello, tampoco mejoran de una manera uniforme, lo que depende del contexto y de la etapa de desarrollo de la escuela en ese proceso (Barber, 2010; Hopkins, 2009).

Por las razones descritas, una evaluación que se oriente a reconocer y a juzgar el mejoramiento educativo, necesariamente obligará a observar procesos, ampliar las fuentes de información y sus métodos de recolección, de manera de brindar la flexibilidad necesaria para evaluar de manera completa y justa este fenómeno. El desarrollo de la investigación y propuestas en esta materia han permitido abrir un camino de desarrollo en el ámbito de la evaluación, pero este se encuentra lejos aún de completarse. A continuación, se identificará un 
conjunto de desafíos que las propuestas metodológicas de evaluación del desempeño de las escuelas debieran tener en consideración, para recoger la perspectiva del mejoramiento educativo.

\section{Consideraciones para una evaluación de desempeño de escuelas vinculada a la complejidad del proceso de mejoramiento}

Asumiendo los elementos discutidos anteriormente, en primer lugar una evaluación del proceso de mejoramiento educativo debiera considerar, tal como indica el propio Levin (2007), la condición sistémica del cambio, es decir, hay un conjunto de elementos que deben combinarse positivamente entre sí para avanzar en el mejoramiento educativo. En la misma línea, Loogma, Tafel-Viia \& Marik (2012) señalan que el mejoramiento educativo no es un proceso lineal, porque normalmente la idea y características de aquello que se busca transformar sufren modificaciones durante el proceso y terminan ocurriendo situaciones diferentes a las planificadas. Esto implica que, junto con el proceso, será necesario observar la presencia de determinados indicadores o atributos clave para constatar la presencia de mejoramiento independiente de las vías por las que se produjo.

MacBeath (2011) explica que en relación con la condición de mejoramiento no hay definiciones únicas, debido a que este se puede considerar como una variable independiente o dependiente, es decir, podría ser el origen o el resultado de iniciativas o procesos de reforma. Además, su contenido va a estar directamente relacionado con el actor educativo en el que se enfoca.

Esta situación implica considerar que la mejora incluye la presencia de transformaciones generadas "desde dentro" de la escuela, relacionadas con el desarrollo de capacidades. Sin embargo, este proceso es apoyado y fomentado "desde fuera", tarea que realizan las políticas educativas y otros organismos. Los atributos del mejoramiento están, entonces, distribuidos en las diferentes capas del sistema educativo considerando una perspectiva multinivel, debido a que implica a estudiantes, padres, docentes, funcionarios 
de la educación, la comunidad, el distrito (o el nivel intermedio) e incluso la cultura.

Como contrapartida, el proceso de mejoramiento ocurre en el marco de un contexto específico de dicho sistema, por lo que su evaluación debiera tener también en cuenta este fenómeno, comprendiendo que las políticas educativas y el contexto afectarán diferenciadamente las tareas más cercanas del centro del proceso.

En relación con la importancia del contexto en la evaluación, Guhn (2009) señala que se ha hecho necesario que las diferencias contextuales y culturales deban estudiarse en profundidad, para poder incorporar esa información en la supervisión de los procesos de implementación reales. El contexto entonces debiera también tenerse en cuenta en la evaluación del proceso de mejoramiento.

Pese a la demanda y a la necesidad de diversificar el espectro de observación del mejoramiento hacia otros eslabones del sistema y el contexto escolar, es importante constatar como contrapartida la idea de que este proceso cuenta con atributos que son relativamente estables. Así, Hallinger \& Heck (2011) analizan un conjunto de elementos que pueden considerarse de manera fundamental en esta materia, indicando que existe un énfasis en aspectos referidos al cambio organizacional y transformaciones en la cultura de las escuelas. Otros dos aspectos relevantes de estudio son el liderazgo educativo y el cambio en las prácticas pedagógicas. También lo es la combinación de estos elementos, dentro de un contexto específico. Por último, este modelo de análisis del mejoramiento educativo se cierra con la variable tiempo, pues se comprende que estos procesos son de carácter longitudinal. De esta manera, una evaluación del proceso de mejoramiento debe ser flexible, pero a la vez no debe olvidar una base de atributos mínimos que lo componen de manera regular.

Estos elementos constitutivos del mejoramiento pueden consolidarse bajo la idea fundamental de desarrollo de capacidades. Para Harris (2012) la principal característica de este concepto está dada por el hecho de que los actores inviertan su energía en hacer cosas diferentes, aprender nuevas destrezas y generar prácticas más 
efectivas. La construcción de capacidades debe ser sistémica, es decir, debe incluir a los diferentes actores y escenarios, para generar una infraestructura que permita cambiar prácticas profesionales. De acuerdo con lo que afirma Harris, el proceso de desarrollo de capacidades puede potenciarse desde afuera y desde adentro. En ambos casos, el elemento clave para que ello ocurra es la colaboración.

Teniendo en consideración los aspectos mencionados de esta revisión bibliográfica, y asumiendo los desafíos de profundizar en la investigación del mejoramiento educativo, desde esta investigación se consideró que la evaluación de desempeño de las escuelas debiera contemplar:

a. Múltiples niveles y dimensiones involucradas en el proceso.

b. Vinculación con el contexto en que se implementa.

c. Diversas fuentes e instrumentos para captar la complejidad.

Junto con lo anterior, bajo la lógica del desarrollo de capacidades, parece ser necesario que se incluya la autoevaluación, como recurso relevante para la utilidad del proceso de evaluación hacia las comunidades que participan de este. De esta forma, el juicio evaluativo es construido a través de la combinación de una mirada externa e interna, como una apuesta para brindar sentido a sus resultados. Así, la estructura y resultados de la evaluación debieran convertirse en un insumo que sea útil para que las escuelas puedan fortalecer sus procesos de mejoramiento.

Finalmente, considerando lo mencionado por MacBeath (2011), en cuanto a su condición de variable independiente o dependiente, esta propuesta se abrió a la posibilidad de no excluir información tanto de procesos como de resultados educativos de la escuela, debido a que en ambos casos se trata de atributos que ayudan a identificar la presencia de los procesos de mejoramiento.

\section{Metodología: elaborando un marco analítico para evaluar los procesos de mejoramiento}

Esta investigación buscó avanzar hacia la evaluación de atributos del mejoramiento educativo en escuelas chilenas, a través de la 
identificación, definición y valoración de las dimensiones y variables que caracterizan este proceso. Para alcanzar este objetivo, se construyó un modelo de análisis a partir del consenso entre actores clave, expertos en este proceso y no únicamente de la recolección de sus opiniones. En este artículo, tal como fue definido al inicio, se abordará el proceso de diseño del modelo de evaluación y de su operacionalización teórica.

En línea con el concepto de mejoramiento educativo, se identificaron cinco tipos de expertos en este proceso, esperando reconocer y aprovechar diferentes tipos de conocimiento en relación con el fenómeno de estudio. Los tipos de expertos se asociaron a procesos centrales referidos al mejoramiento:

a. Protagonizar el mejoramiento educativo, es decir, trabajar directamente en los procesos de enseñanza y aprendizaje (docentes).

b. Gestionar el mejoramiento educativo, a partir de la organización del trabajo educativo en la escuela (equipo directivo).

c. Apoyar el mejoramiento educativo, recogiendo la experiencia de los asesores educativos de las escuelas.

d. Estudiar el mejoramiento educativo, lo que implica considerar la reflexión proveniente de investigadores que estudien este fenómeno.

e. Incidir políticamente en el mejoramiento educativo, considerando a quienes están cerca de la toma de decisiones en el ámbito de las políticas educativas.

Se consideraron, combinaron y sintetizaron estos cinco saberes en el modelo de evaluación a través de la metodología Proceso Analítico Jerárquico (AHP, por sus siglas en inglés) (Saaty, 1997). El AHP tiene por objeto sintetizar un conjunto de factores y valores en un modelo tipo árbol que, de manera sistémica, componen un determinado concepto u objeto de evaluación. Su estructura se basa en la descomposición de estructuras complejas en sus componentes de manera jerárquica. Para ello, se asignan valores numéricos a las definiciones y valoraciones realizadas por los actores expertos, ayudando a definir la importancia relativa de cada componente en la 
explicación del fenómeno. La metodología considera el cumplimiento de tres principios: construcción de jerarquía, asignación de prioridades y consistencia lógica.

La construcción de la jerarquía implica la elaboración del modelo general de evaluación del objeto y su descomposición en dimensiones y criterios. Este proceso se desarrolla con la participación de los actores expertos, que normalmente tienen discursos y valoraciones disímiles. Por esto, se requiere el logro de consensos fundamentales entre ellos, satisfaciendo un conjunto de requerimientos de validez, asociados a cuatro axiomas: reciprocidad, homogeneidad, dependencia y expectativas (Saaty, 1994). El segundo principio -la asignación de prioridades-implica pedir a los expertos que asignen juicios al comparar pares de elementos (llamados "criterios" o "subcriterios") semejantes en cada nivel de una jerarquía, en relación con un criterio del nivel inmediatamente superior. La pregunta clave que deberá responderse es “¿Cuánto más importante o preferible es un criterio sobre otro del mismo nivel, respecto del criterio del nivel inmediatamente superior?". A partir de dichas prioridades se define una escala, cuyos valores se registran en una matriz cuadrada. El vector propio principal de la matriz representa la síntesis de los juicios de los expertos, que a su vez permite obtener las prioridades de los criterios o subcriterios que conforman la estructura jerárquica, considerando un nivel tolerable de inconsistencia entre las valoraciones. Por último, el tercer principio del AHP es la consistencia lógica, la que tiene relación con el grado de dispersión de los juicios de valor emitidos por los diferentes actores y el cumplimiento de parámetros lógicos de organización de las prioridades. Para ello, el AHP provee una teoría para revisar la inconsistencia de estos juicios, la que no debe superar el 10\% (Saaty, 1997). El siguiente esquema describe la estructura evaluativa con la metodología AHP. 
Figura 1: Estructura jerárquica de la metodología multicriterio, AHP

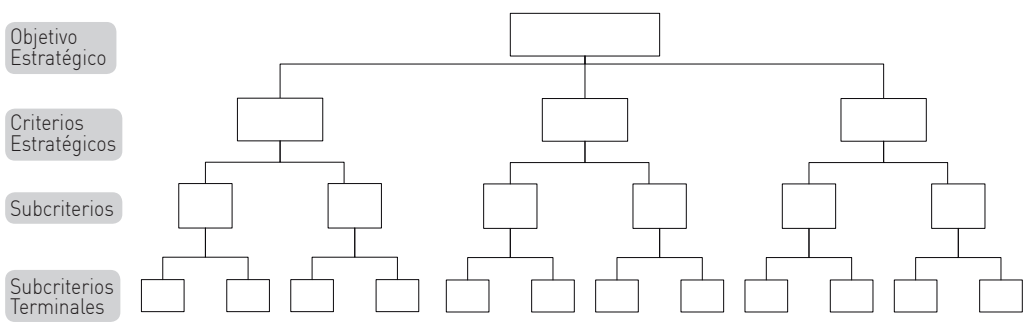

\section{Método AHP aplicado al mejoramiento educativo}

Considerando la metodología descrita, a continuación se describe el diseño implementado en esta investigación.

En primer lugar, se construyó un documento base de la discusión acerca del mejoramiento educativo a nivel de establecimientos educacionales, a partir de la revisión de la literatura nacional e internacional. Este documento fue discutido de manera separada por los siguientes cuatro grupos de expertos ya identificados: docentes, directivos, asesores técnico-educativos (ATE) e investigadores.

Los participantes fueron escogidos a través de la siguiente metodología. En el caso de los directores y docentes, se les escogió considerando que su establecimiento -siendo municipal o particular subvencionado- haya tenido dos alzas continuas en su promedio SIMCE de Lenguaje y Comunicación en los últimos tres años. Respecto de las ATE, se seleccionó a instituciones que brindaran asesoría a nivel nacional con sostenedores que administren sobre 20 establecimientos y que contaran con una presencia de, al menos, cinco años en asesoría, que les permitiera identificar un conocimiento de diferentes grupos de escuela. Finalmente, en relación con los investigadores se realizó una revisión de los grupos e instituciones que tenían en marcha procesos indagatorios referidos al mejoramiento educativo en el momento de la investigación, y que provinieran de diferentes instituciones. Todos los participantes estaban localizados en la Región Metropolitana.

Una primera etapa del trabajo de campo consistió en la realización de focus group con los cuatro grupos mencionados. A ellos 
se les entregó el documento base y se les pidió que -una vez leído el documento- fueran identificando y describiendo cuáles de los elementos descritos - u otros- caracterizan a una escuela chilena que está mejorando educativamente y de qué forma ocurre ello. Al final de la sesión, se presentó una gráfica que sintetizaba los principales elementos surgidos en la discusión para la validación de los asistentes.

La información recopilada en los focus group fue sistematizada y analizada. Luego, se avanzó hacia su síntesis en una primera propuesta de matriz jerárquica AHP, que permitiera incorporar los cuatro resultados obtenidos. Para avanzar en este proceso fue importante consignar que entre los grupos de expertos se observaron importantes semejanzas en cuanto a la identificación de criterios que caracterizaban el mejoramiento educativo y su valoración. De hecho, no se observaron grandes contradicciones en esta materia entre los participantes.

La segunda etapa del trabajo se inició con la validación de la estructura jerárquica. Para ello, se planificó una reunión individual con un representante de cada grupo de expertos, quien actuaría como "vocero" de su grupo, validando la propuesta de matriz según la discusión establecida en los focus group.

La tercera etapa de trabajo consistió en el desarrollo de un taller en el que participaron los mismos voceros, más un representante de la institucionalidad educativa (Agencia de Calidad de la Educación). Este representante se incluyó en esta etapa porque la metodología indica que la estructura jerárquica también debe tener un criterio de factibilidad de aplicación, vinculada con quienes están en la toma de decisiones, a fin de que estos opinen si es posible que la metodología se aplique.

En esta jornada se realizaron las siguientes acciones: validar la estructura jerárquica, cumpliendo con los cuatro axiomas definidos por el método y establecer las prioridades entre los criterios identificados. Esta tarea se realizó con el apoyo de un software especializado para la metodología AHP, denominado Expert Choice. De esta forma, se contó con la estructura jerárquica definitiva que da cuenta de la presencia de procesos de mejoramiento en escuelas chilenas, de acuerdo con los expertos. 
Finalmente, la cuarta parte del proceso consistió en que, sobre la base de los acuerdos alcanzados en el momento anterior, se realizó la definición de los indicadores que permitirán el levantamiento de información respecto de los atributos de mejoramiento educativo descritos por cada criterio terminal de la estructura jerárquica.

Posteriormente, con base en los resultados recién descritos, se realizó el diseño de los instrumentos de recolección de información y/o la identificación de datos secundarios que permitirán levantar los antecedentes para informar a cada indicador de la estructura jerárquica. Luego, esos instrumentos fueron sometidos a una evaluación de validez de contenido. Para ello, se les solicitó a dos jueces expertos analizar el instrumental generado, produciendo con ellos ciclos iterativos que permitieron lograr el estándar de calidad idóneo en cuanto a validez.

La primera etapa de esta investigación, cuyos resultados se reportan a continuación, finalizó con la última acción mencionada. De esta forma, se detallará en seguida tanto la estructura del modelo evaluativo, como la propuesta metodológica para su implementación y la discusión de sus fortalezas y debilidades desde un punto de vista teórico, previamente a su aplicación en escuelas.

\section{Estructura jerárquica para la evaluación de atributos del mejoramiento educativo}

El resultado del proceso descrito fue una matriz jerárquica que desglosa en criterios el objetivo de medir la presencia de atributos de mejoramiento educativo en escuelas chilenas. Esta matriz se describe a continuación, junto con el valor de prioridad que les asignaron los expertos.

La matriz se organiza en tres criterios estratégicos, de los que se desprende un conjunto de subcriterios o atributos del mejoramiento educativo. Estos tres criterios son:

a. Impulsos externos al mejoramiento, es decir, la forma en que la escuela utiliza y aprovecha insumos y recursos provenientes de 
74 HACIA UN MARCO ANALÍTICO Y METODOLÓGICO PARA LA EVALUACIÓN DEL MEJORAMIENTO EDUCATIVO EN ESCUELAS CHILENAS - L. De la Vega

la política educativa y otros agentes.

b. Gestión del sostenedor orientada al mejoramiento, lo que implica la eficiencia de la gestión del sostenedor y su liderazgo pedagógico.

c. Movilización interna del mejoramiento, es decir, de qué forma la escuela es capaz de definir y asumir un proceso de cambio que tenga sentido con su historia, desafíos y necesidades. 


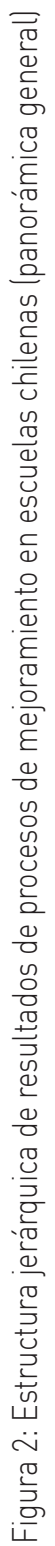

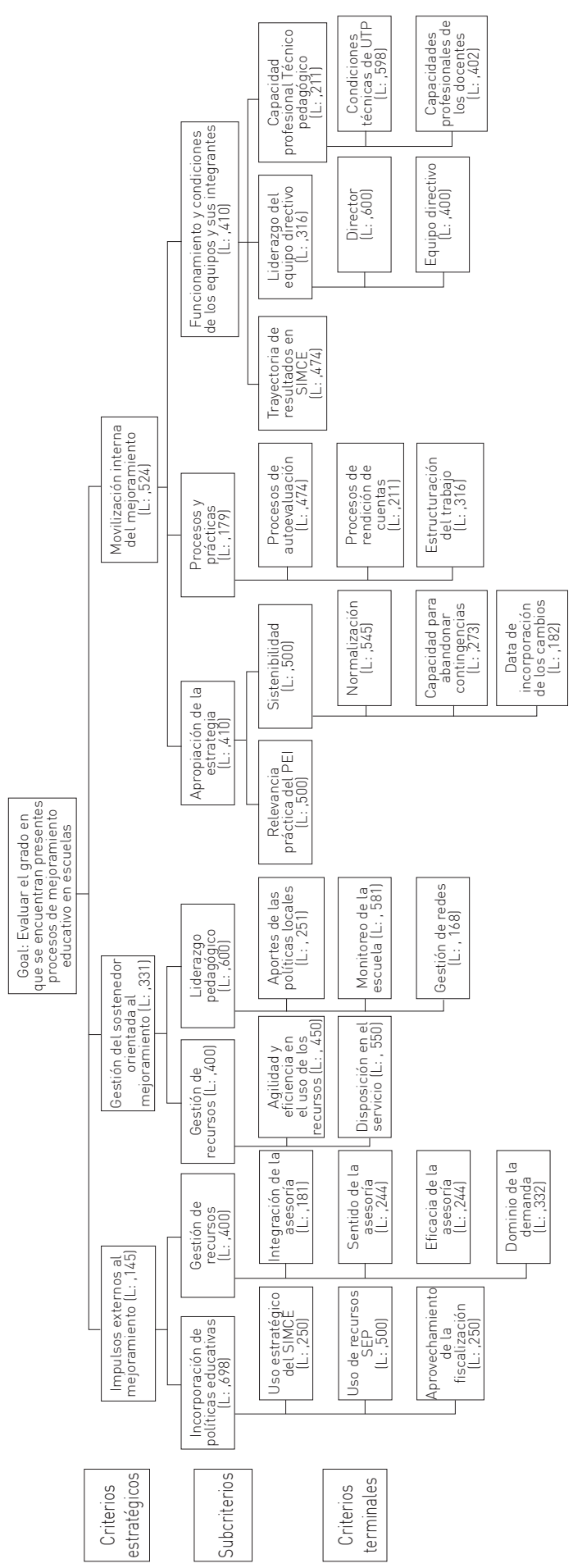


Figura 3: Estructura jerárquica. Criterio estratégico "Impulsos externos al mejoramiento"

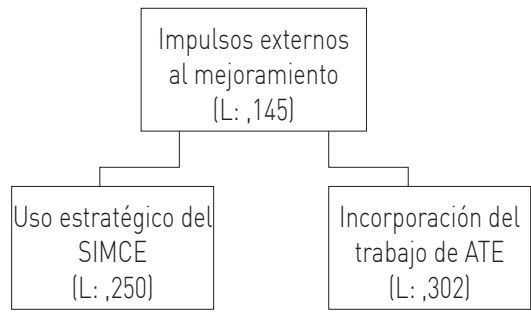

Uso de recursos
SEP
$(L:, 500)$

Aprovechamiento de la fiscalización (L: , 250)

$$
\begin{gathered}
\text { Integración de la } \\
\text { asesoría } \\
\text { (L: , 181) }
\end{gathered}
$$

\begin{tabular}{|c|}
\hline Sentido de la \\
asesoría \\
(L: ,244)
\end{tabular}

\begin{tabular}{|c|}
\hline Eficacia de la \\
asesoría \\
(L: , 244)
\end{tabular}

Dominio de la
demanda
(L: ,332)

Figura 4: Estructura jerárquica. Criterio estratégico "Gestión del sostenedor orientada al mejoramiento"

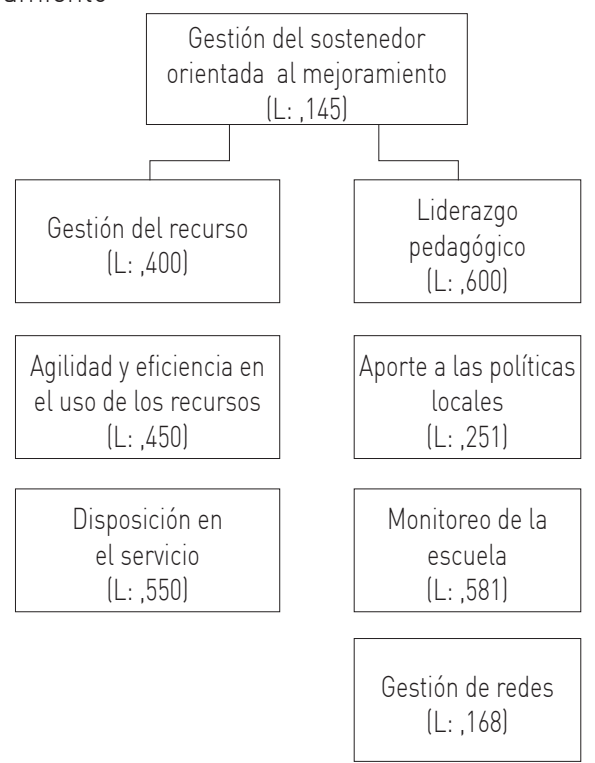




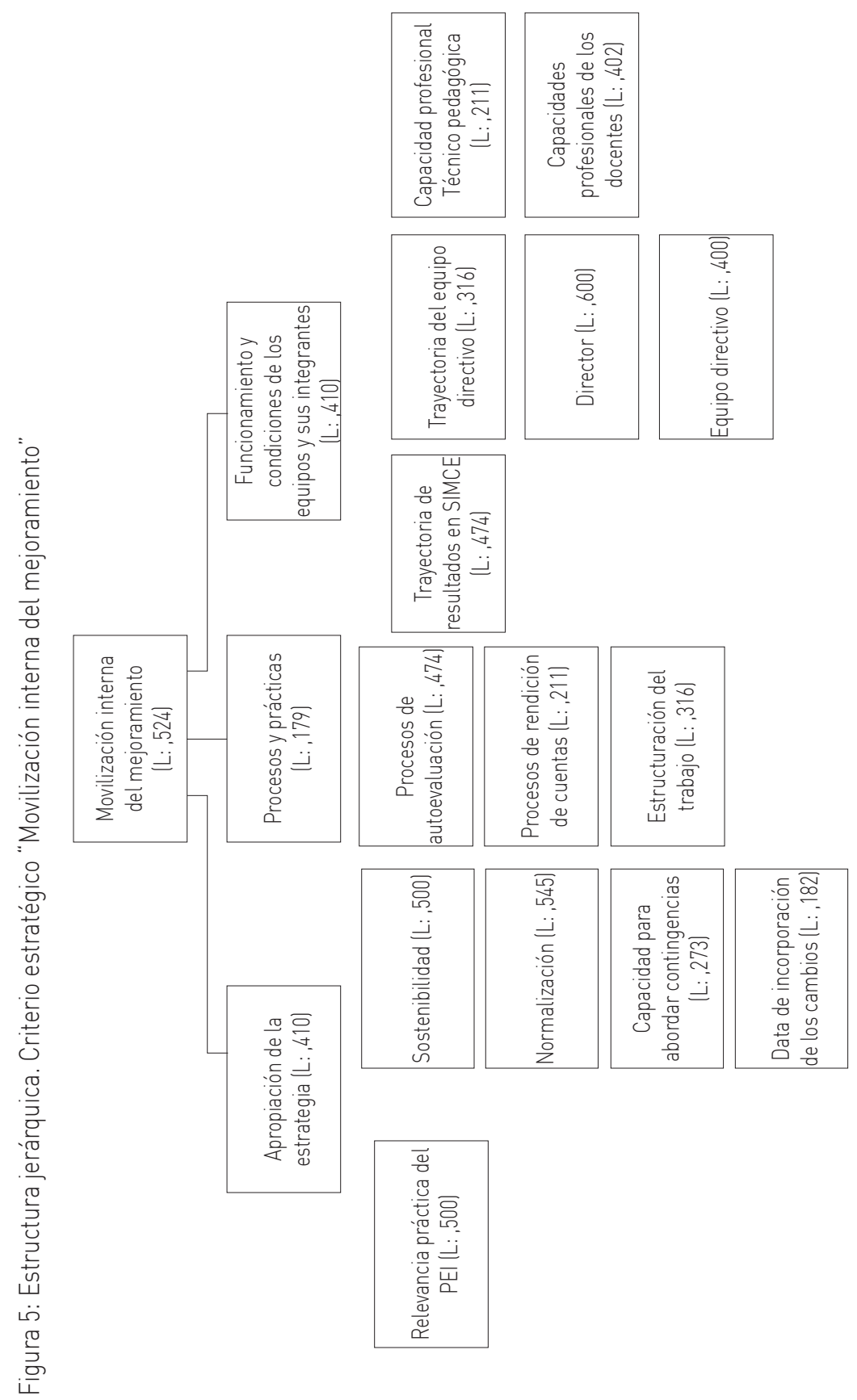




\section{Descripción de resultados del diseño del modelo jerárquico para la evaluación}

Tal como puede observarse en las figuras presentadas, de cada uno de los tres criterios estratégicos se desglosó un conjunto de subcriterios y criterios terminales o indicadores. Estos recogen diferentes procesos, prácticas y resultados que fueron reconocidos como atributos de una escuela que se encuentra en un proceso de mejoramiento. Los expertos entregaron mayor relevancia al tercer criterio estratégico (Movilización interna del mejoramiento), por lo que dotaron a sus atributos de una mayor ponderación en la evaluación. Posteriormente, se calificó en segundo lugar al criterio de Aporte del sostenedor y finalmente a los Impulsos externos al mejoramiento.

En la misma línea, en el proceso realizado se destacó con claridad el concepto de liderazgo pedagógico, como componente central de conducción de los procesos de cambio en la escuela. Este factor es el único que se menciona en dos ocasiones (a nivel de sostenedor y dentro de la escuela). Junto con ello, se observó un consenso en que el liderazgo es más bien un atributo o función de las instituciones educativas y que este descansa más allá de la figura de su director. Esto implica considerar la relevancia del análisis de la distribución del liderazgo, a la hora de evaluar las características de los procesos de mejoramiento (Harris \& Spillane, 2008).

Junto con lo anterior, se identifican en las políticas educativas insumos muy importantes para dar impulso a los procesos de mejoramiento, y se reconoce que ellos gatillan cambios, pero que el protagonismo para que se realicen recae sobre todo en la escuela.

Si se revisa en detalle el conjunto de subcriterios que dan cuenta de la estructura jerárquica, los componentes de los procesos de mejoramiento son fundamentalmente de dos tipos: el primero se refiere a condiciones de funcionamiento de la escuela y apunta a la estructura que soporta los procesos de mejoramiento (normalización en la escuela, gestión eficiente de los recursos, o estructuración del trabajo, entre otros). Por su parte, el segundo tipo alude al desarrollo de competencias individuales, colectivas y organizacionales (enseñanza, procesos de autoevaluación o uso de información). 
Teniendo en consideración las expectativas definidas para esta propuesta y la estructura jerárquica recién presentada, la evaluación de los atributos de mejoramiento educativo en las escuelas buscará constatar la presencia de los distintos indicadores que lo componen. Bajo esta lógica, una escuela podrá contar con una mayor o menor presencia de procesos de mejoramiento en relación con otras escuelas, considerando un continuo entre 0 (sin presencia de atributos de mejoramiento) y 1 (con total presencia de estos atributos). Por su parte, esta estructura también servirá para evaluar este resultado en distintos momentos del tiempo, lo que favorecerá el reconocimiento de mejoramiento o empeoramiento de la presencia de atributos de mejoramiento de la escuela.

Es decir, la lógica de la evaluación no identifica por ahora un estándar de logro, sino que su resultado tiene relevancia en relación con el resultado obtenido por otra escuela, o por ella misma, en otro momento del tiempo.

\section{Diseño de indicadores e instrumentos de recolección de información e identificación de datos secundarios}

Con posterioridad a la validación de la estructura jerárquica para evaluar la presencia de atributos de mejoramiento, se avanzó hacia la última etapa del trabajo, correspondiente a la identificación de los indicadores necesarios para implementar una evaluación de cada uno de los criterios terminales de la estructura jerárquica, así como del reconocimiento de la información necesaria para los indicadores.

Cada uno de estos indicadores fue validado por cada vocero experto, en una reunión personalizada, recogiendo e incorporando los cambios y sugerencias emitidos por estos. Todo el proceso descrito fue apoyado y validado por una académica experta en la metodología AHP.

Teniendo en consideración las definiciones realizadas en la sección conceptual de este trabajo, el diseño de los indicadores para la evaluación de atributos de mejoramiento en los establecimientos 
educacionales buscó favorecer una mirada amplia y diversa respecto de los atributos del mejoramiento educativo, para contar con un panorama más justo acerca del trabajo que se lleva a cabo en los establecimientos educacionales. De esta forma, para cada criterio terminal se irá justificando la decisión de la metodología e instrumento que se aplicará para la evaluación, considerando criterios metodológicos, educacionales y prácticos.

La siguiente tabla sintetiza las definiciones realizadas en materia de recolección de información para cada indicador desprendido de la matriz jerárquica. 


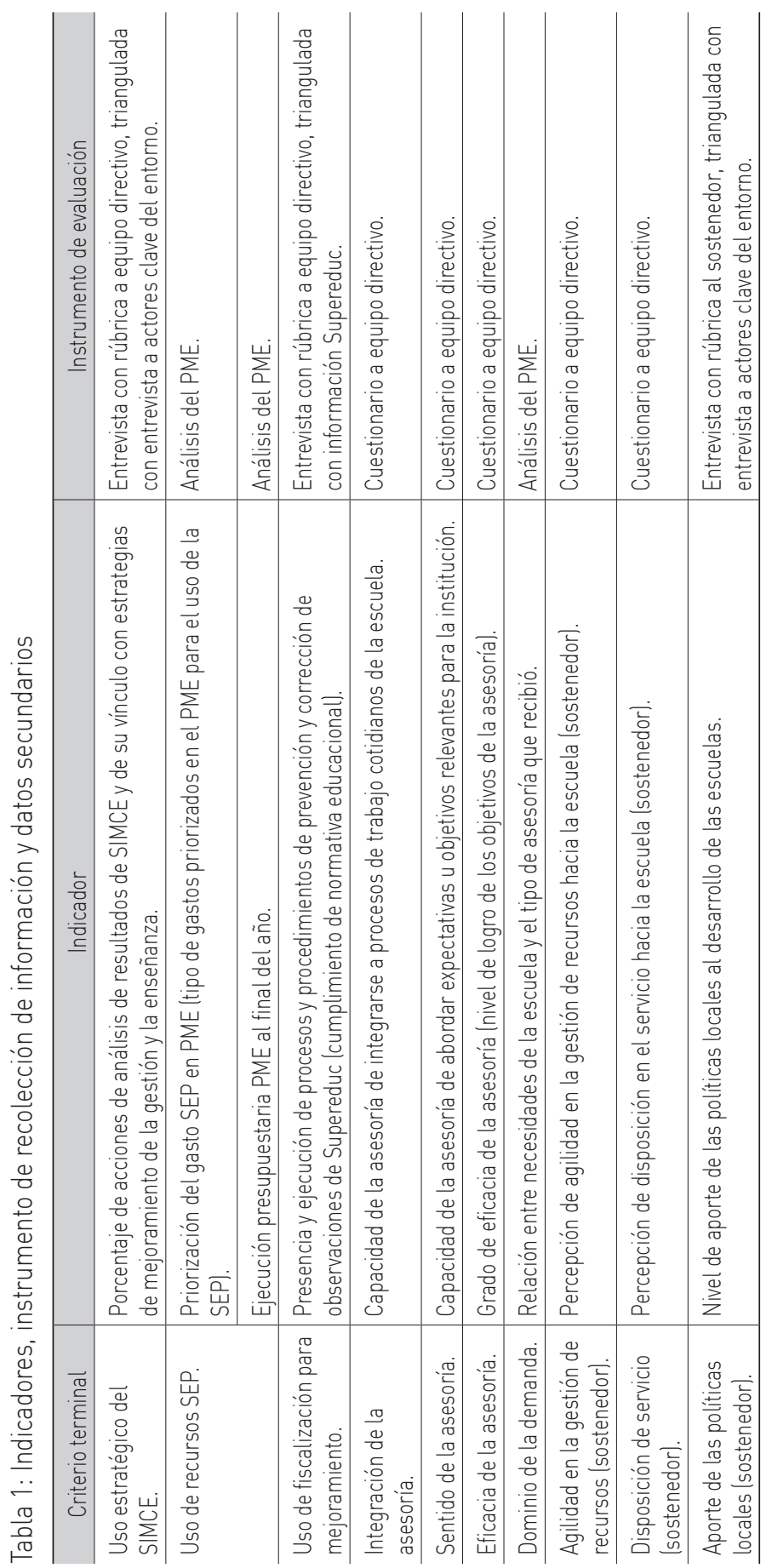




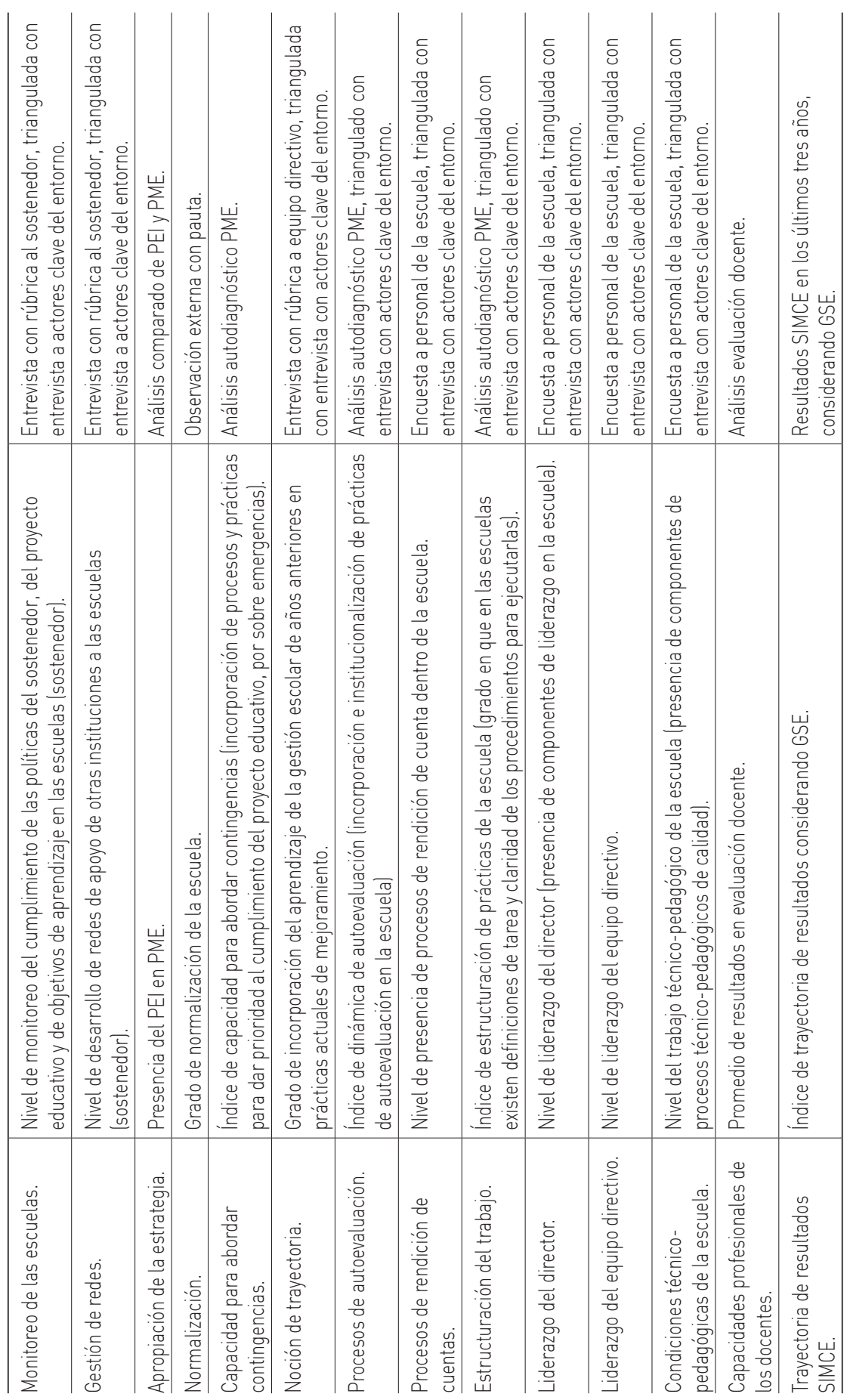




\section{La incorporación del contexto en la evaluación}

Thrupp (1998) es un investigador que en las últimas décadas se ha destacado por poner en cuestionamiento las limitaciones de la no consideración del contexto social en el análisis del logro de las escuelas. Según indica, no es posible escindir a la escuela de su contexto, porque es este el que define fundamentalmente sus características y condiciones de trabajo.

En otro texto, Thrupp y Lupton (2006) profundizan en esa reflexión, a través del uso de evidencia de investigaciones que describen y demuestran el impacto del contexto social en los procesos y resultados de los estudiantes, incluso entre escuelas de similar nivel socioeconómico. Estas afirmaciones son una base para reconocer y definir la manera en que el contexto debiera ser considerado en la evaluación del mejoramiento educativo. Al respecto, Thomas (2010) estima que es pertinente que el juicio sobre el mejoramiento de los resultados esté acompañado por antecedentes tales como el tiempo, procesos desarrollados en la escuela, la composición de este en términos del tipo de alumnos que la integran y el contexto socioeconómico en que se desarrolla el trabajo de la escuela. Sin embargo, según señala el propio Thrupp y sus colegas (Thrupp, Lupton \& Brown, 2007), no se trata de llegar a esperar que cada contexto sea un mundo diferente, imposible de comparar, sino más bien aceptar que hay comunidades o grupos que son socialmente desventajados y que es necesario reconocer esto, tanto en la investigación, como a nivel social y político.

Teniendo estos elementos en cuenta y considerando los resultados del modelo de evaluación descritos, se propone que la matriz jerárquica establezca diferente ponderación general de acuerdo con dos variables principales: grado de vulnerabilidad socioeconómica y estado de desarrollo educativo de la escuela. De esta forma, la valoración de atributos de mejoramiento debiera reconocer aspectos que hacen más difícil el avance en el logro de una evaluación positiva o favorable.

Sin embargo, la definición recién establecida podría haberse hecho sin contar con los resultados de esta investigación. Por esta 
razón, y teniendo en cuenta el foco del mejoramiento en el núcleo pedagógico y los resultados de la matriz consensuada por los expertos, se propone que las variables más estrechamente asociadas al núcleo pedagógico tengan una ponderación diferenciada, dependiendo del grado de vulnerabilidad socioeconómica -dada la composición de la escuela-y su nivel de desarrollo durante su proceso de mejoramiento. Así, los indicadores correspondientes al criterio estratégico de "Movilización interna del mejoramiento" serían los que tendrían esta ponderación diferenciada (en este caso de menor valor en la medida en que aumente el grado de vulnerabilidad socioeconómica), que reconozca la mayor dificultad de las escuelas que dificultades socioeconómica mayores y mejor desarrollo educativo. La única excepción se hace en el caso de indicador "Normalización", debido a que corresponde a la existencia de procesos básicos de funcionamiento de la escuela.

\section{Discusión}

Este artículo ha buscado destacar de manera concreta las potencialidades que una innovación metodológica puede prestar al análisis del mejoramiento educativo. Se considera que la experiencia que estudia esta investigación es innovadora, debido a que tiene como objetivo que se reconozcan de manera amplia diferentes tipos de atributos del mejoramiento educativo para llevar a cabo evaluaciones más justas. Además, estos atributos tuvieron una expresión concreta en instrumentos de recolección de información, identificación de datos o la combinación de ambos, como estrategias concretas de abordar la evaluación. Es decir, no hubo indicadores que no pudiesen traducirse en algún mecanismo que permitiera su evaluación, pese a que es importante reconocer que algunos de ellos son de compleja aplicación.

Junto con esto, la técnica de recolección de información para el diseño del modelo de evaluación no incluyó únicamente el levantamiento de información de parte de actores, sino que consideró la tarea de que estos tomen acuerdos concretos en relación con los componentes de la estructura jerárquica y la relevancia relativa de cada uno de sus componentes. El ejercicio de búsqueda de consenso es el que explica que se haya optado por un número de integrantes 
algo más acotado que si se hubiese esperado únicamente recoger información proporcionada por estos participantes. De acuerdo con los resultados obtenidos, puede concluirse que la apuesta por lograr consensos evaluativos fue exitosa.

Se considera que en general la apuesta metodológica descrita favoreció el cumplimiento de la etapa relatada en este artículo. En primer lugar, ha sido posible vislumbrar, luego de la implementación de la investigación y el levantamiento de los criterios de evaluación, la presencia de aspectos comunes entre el escenario chileno y la investigación referida al mejoramiento educativo en la investigación educacional, especialmente la relevancia de la apropiación de estos procesos de parte de la escuela. Con ello se confirma la importancia de privilegiar las características de los procesos internos de los establecimientos educacionales a la hora de evaluar su desempeño.

Al mismo tiempo, ha sido posible reconocer elementos propios de la realidad chilena en la identificación de procesos de mejoramiento. Aquí resulta relevante la identificación de la labor del sostenedor como factor clave para la implementación de procesos de mejoramiento, reconociendo aspectos de gestión de recursos y sobre todo de liderazgo pedagógico.

La investigación permitió constatar de manera teórica la importancia de contextualizar el uso de estos indicadores en la evaluación. En la discusión de los expertos se destacó que el conjunto de indicadores debiera considerar el contexto social en que se encuentra la escuela, como un factor ponderador de los resultados de la evaluación. Además, el uso de la revisión bibliográfica permitió desarrollar una propuesta concreta de corrección en la estructura jerárquica, considerando el grado de vulnerabilidad de la escuela y su grado de desarrollo educativo. Debido a las características de la investigación desarrollada, no fue posible identificar ni valorar en qué medida cuantitativa la estructura jerárquica de evaluación debiera adaptarse a las diferencias del contexto en el que opera la institución educativa.

Este elemento se considera una limitación de la investigación que hace necesario el ejercicio de aplicar la metodología definida, 
para poder valorar de manera clara en qué medida se incorpora este importante factor en el análisis.

En otro ámbito de análisis, la comparación de los estándares indicativos que utiliza la Agencia de Calidad de la Educación en sus visitas de evaluación, respecto de los resultados de esta investigación muestra intersecciones, pero también elementos que no son comunes. Esta situación implica un amplio margen de desarrollo para incorporar los atributos de mejoramiento en la evaluación de escuelas chilenas. Los resultados de la aplicación de la propuesta de evaluación también pueden ayudar a los procesos de autoevaluación y reflexión que dicha agencia propone a los establecimientos y favorecer la toma de decisiones para la mejora.

De la revisión ex post del diseño de los indicadores e instrumentos ha sido posible identificar un conjunto de aspectos que en la metodología diseñada no favorecen el logro completo del análisis de resultados de procesos de mejoramiento y que debieran ser estudiados con detalle para avanzar en su resolución. Estos elementos se relacionan con el levantamiento de información cualitativa que favorezca la identificación más fina de procesos involucrados en algunos de los criterios terminales que han sido diseñados.

En concreto, algunos criterios terminales, junto con los instrumentos diseñados, podrían ser complementados si es que se incluyen elementos adicionales de registro o sistematización de información. Ejemplo de ello son el ámbito de uso de los resultados del SIMCE o la fiscalización de la Superintendencia, por un lado, y elementos relacionados con la instalación de determinadas prácticas dentro de las escuelas, por otro. En este tipo de criterios terminales se ha sugerido generar información complementaria que permita una mayor comprensión de los fenómenos, más allá de la identificación de la presencia o ausencia de atributos.

Considerando lo anterior, se vislumbra un área de mejoramiento para la propuesta desarrollada, orientada a la tarea de generar instrumentos de carácter cualitativo para complementar algunos de los indicadores. Sin embargo, es necesario señalar que antes de ello es 
necesario desarrollar una primera aplicación del instrumento, donde sea posible constatar esta limitación u otra.

Es útil recalcar a modo de reflexión que los instrumentos de evaluación, sobre todo de fenómenos complejos como el mejoramiento educativo, son objetos en desarrollo que requieren de una revisión permanente y colectiva, de manera de ser periódicamente perfeccionados sobre la base de la evidencia empírica de su aplicación. Además, por su misma naturaleza, son construcciones en las que difícilmente se logra consenso completo y que están expuestas a la identificación de debilidades o carencias. Todos estos aspectos son importantes desafíos para el desarrollo de este mecanismo de evaluación y para otros en general.

Finalmente, se debe señalar que las limitaciones descritas se convierten en tareas que debieran ser consideradas en la segunda etapa de este programa de investigación. Esta tendrá como objetivo la validación empírica de la propuesta metodológica que ha sido diseñada, a través de su aplicación en establecimientos educacionales de diferente contexto social y educativo, siguiendo la propuesta para considerar diferenciadamente el contexto en la evaluación. El análisis de los resultados de la aplicación permitirá profundizar en el modelo, clasificando sus variables desde un punto de vista empírico, identificando su condición de variable independiente, dependiente o moderadora del mejoramiento educativo.

Junto con ello, será el momento para evaluar la idoneidad de las definiciones de instrumentos de recolección de información y/o datos que se consideraron en esta primera versión del trabajo. Por último, se espera además avanzar en un mecanismo productivo de devolución de la información a los establecimientos educacionales que participen del proceso y que les permitan extraer información o sugerencias para la implementación de estrategias de mejoramiento. 


\section{Referencias}

Anderson, S., Mascall, B., Stiegelbauer, S. \& Park, J. (2012). No one way: differentiating school district leadership and support for school improvement. Journal of Educational Change, 13, 403- 430.

Barber, M. (2010). How government, professions and citizens combine to drive successful educational change. En A. Hargreaves, A. Lieberman, M. Fullan, D. Hopkins (Eds.), Second international handbook of educational change (pp. 261 - 278). Dordrecht Heidelberg Londres, Nueva York: Springer Science+Business Media.

Bellei, C., Valenzuela, J. P. y De los Ríos, D. (2010). Segregación escolar en Chile. En S. M. y G. Elacqua (Eds.), Fin de ciclo: cambios en la gobernanza del sistema educativo (pp. 209- 229). Santiago: Facultad de Educación, Pontificia Universidad Católica de Chile y Oficina Regional para América Latina y el Caribe Unesco.

Bogotch, I., Mirón, L. \& Biesta, G. (2007). "Effective for what; effective for whom?" Two questions SESI should not ignore. En T. Townsend (Ed.), International Handbook of School Effectiveness and Improvement (pp. 93110). New York: Springer.

Bourke, R. \& Mc Gee, A. (2012). The challenge of change: using activity theory to understand a cultural innovation. Journal of Educational Change, 13, 217-233.

Chrispeels, J. \& González, M. (2006). The challenge of systemic change in complex educational systems. A district model to scale up reform. En A. Harris \& J. Chrispeels (Eds.), Improving schools and educational systems. International perspectives (pp. 242 - 263). Nueva York: Routledge.

Dale, R. \& Robertson, S. (2007). Beyond methodological 'isms' in comparative education in an era of globalization. En A. Kazamias \& R. Cowan (Eds.) Handbook on Comparative Education. Netherlands: Springer.

Datnow, A. \& Park, V. (2004). Conceptualizing policy implementation: Largescale reform in an era of complexity. En G. Sykes, \& P. Schneider (Eds.), Handbook of educational policy research. Nueva York: American Educational Research Association.

Datnow, A. (2012). Collaboration and contrived collegiality: Revisiting Hargreaves in the age of accountability. Journal of Educational Change, 12, 147- 158 .

Elmore, R. (2010). Mejorando la escuela desde la sala de clases. (C. Santa Cruz, Trans.). Santiago: Fundación Chile, Fundación CAP.

Froemel, J. (2003). Evaluación de la calidad de la educación con equidad: el modelo de valor agregado. Persona y Sociedad, XVII(1), 165-178. 
Fullan, M. (1982). The meaning of educational change. Nueva York: Teachers College Press.

Fullan, M. (2002). Los nuevos significados del cambio en la educación. Barcelona: Octaedro.

Fullan, M. (2010). Positive pressure. En A. Hargreaves, A. Lieberman, M. Fullan \& D. Hopkins (Eds.), Second international handbook of educational change (pp. 119-130). Dordrecht: Springer Science+Business Media.

Fullan, M. \& Hargreaves, A. (2013). Professional capital: transforming teaching in every school. Nueva York: Teachers College Press.

Guhn, M. (2009). Insights from successful and unsuccessful implementations of school reforms programs. Journal of Educational Change, 10, 337-363.

Hallinger, P. \& Heck, R. (2011). Exploring the journey of school improvement: Classifying and analyzing patterns of change in school improvement processes and learning outcomes, school effectiveness and school improvement. International Journal of Research, Policy and Practice, 22(1), 1-27.

Hamilton, L., Stecher, B. \& Yuan, K. (2012). Standards-based accountability in the United States: lessons learned and future directions. Educational Inquiry, 3(2), 149-170.

Hargreaves, A. (2003). Enseñar en la sociedad del conocimiento. Barcelona: Octaedro.

Hargreaves, A. (2010). Change from without: Lessons from other countries, systems, and sectors. En A. Hargreaves, A. Lieberman, M. Fullan \& D. Hopkins (Eds.), Second international handbook of educational change (pp. 105-117). Dordrecht: Springer Science+Business Media.

Hargreaves, A. (2012). Singapore: The fourth way in action? Educational Research for Policy and Practice, 11(1), 7-17. http://dx.doc.org/ 10.1007/ s10671-011-9125-6

Hargreaves, A. y Fink, D. (2006). Estrategias de cambio y mejora en educación caracterizadas por su relevancia, difusión y continuidad en el tiempo. Revista de Educación, 339, 43-58.

Harris, A. \& Spillane, J. (2008). Distributed leadership through the looking glass. Management in Education, 22(1), 31-34.

Harris, A. (2010). Improving schools in challenging context. En A. Hargreaves, A. Lieberman, M. Fullan \& D. Hopkins (Eds.), Second International Handbook of Educational Change (pp. 105-117). Dordrecht: Springer Science+Business Media. 
Harris, A. (2012). Liderazgo y desarrollo de capacidades en la escuela. (C. Santa Cruz, Trans.). Santiago: Fundación Chile, Fundación CAP.

Hopkins, D. (2009). Hacia una buena escuela: experiencias y lecciones. (C. Santa Cruz, Trans.). Santiago: Fundación Chile, Fundación CAP.

Hopkins, D. (2010). Every school a great school - realizing the potential of system leadership. En A. Hargreaves, A. Lieberman, M. Fullan \& D. Hopkins (Eds.), Second international handbook of educational change (pp. 741-764). Dordrecht: Springer Science+Business Media.

Levin, B. (2007). Sustainable, large-scale education renewal. Journal of Educational Change, 8, 323-336.

Levin, B. (2009). Does politics help or hinder education change? Journal of Educational Change, 10, 69- 72.

Loogma, K., Tafel-Viia, K. \& Marik, M. (2012). Conceptualizing educational changes: A social innovation approach. Journal of Educational Change. http://dx.doi.org/0.1007/s10833-012-9205-2

Martinic, S.; Elacqua, G. (Eds.) (2010). ¿Fin de ciclo? Cambios de gobernanza en el sistema educativo. Santiago. Unesco- P. Universidad Católica de Chile.

Mcbeath, J. (2011). Liderar el aprendizaje dentro y fuera de la escuela (C. Santa Cruz, Trans.). Santiago: Fundación Chile, Fundación CAP.

Mourshed, M., Chijioke, C. \& Barber, M. (2010). How the world's most improved school systems keep getting better. En M. Mourshed, C. Chijioke, \& M. Barber (Eds.) How the world's most improved school systems keep getting better. Londres: McKinsey and Company.

Murillo, F. (2003). El movimiento teórico-práctico de mejora en la escuela. Algunas lecciones aprendidas para transformar los centros docentes. REICE, 1(2), 1- 22.

Murillo, F. y Krichesky, G. (2012). El proceso de cambio escolar. Una guía para impulsar y sostener la mejora de las escuelas. Revista Iberoamericana sobre Calidad, Eficacia y Cambio en Educación, 10(1), Pp. 27- 43.

$\mathrm{Ng}$, P. T. (2012). An examination on school accountability from the perspective of Singapore school leaders. Educational Research Policy and Practice, 12 (2), 121-131.

Organisation for Economic Co-operation and Development, OECD. (2013). Synergies for better learning. An international perspective on evaluation and assessment. OCDE Reviews of Evaluation and Assessment in Education. Paris: OECD Publishing. 
Shwartz, H., Hamilton, L., Stecher, B. \& Steele, J. (2011). Expanded measures of school performance. RAND Corporation. Preparado por Sandler Foundation. Recuperado de http://www.rand.org/pubs/technical_ reports/TR968.html

Saaty, T. (1994). Fundamentals of decision making and priority theory with the Analytic Hierarchy Process. Pittsburgh: RWS Publications.

Saaty, T. (1997). Toma de decisiones para líderes. El Proceso Analítico Jerárquico, la toma de decisiones en un mundo complejo. Pittsburgh: RWS Publications.

San Martín, E. y Carrasco, A. (2012). Clasificación de escuelas en la nueva institucionalidad educativa: contribución de los modelos de valor agregado para una responsabilización justa. Temas de la Agenda Pública del Centro de Políticas Públicas de la Universidad Católica de Chile, No 53.

Thomas, S. (2010). Assessment and the evaluation of institutional effectiveness. International Encyclopedia of Education, 3, 172- 180.

Thrupp, M. (1998). The art of possible: Organizing and managing high and low socioeconomic schools. Journal of Education Policy, 12(2), Pp. 197-219.

Thrupp, M. \& Lupton, R. (2006). Taking school context more seriously: the social justice challenge. Journal of Educational Studies, 54(3), 308-328.

Thrupp, M., Lupton, R. \& Brown, C. (2007). Pursuing the contextualization agenda: Recent progress and future prospects. En T. Townsend (Ed.), International handbook of school effectiveness and improvement (pp. 111126). Dordrecht: Springer.

Vásquez, J, \& Darling-Hammond, L. (2008). Accountability Texas-Style: The progress and learning of urban minority students in a high-stakes testing context. Educational Evaluation and Policy Analysis, 30(2), 75-110.

Weinstein, J. y Muñoz, G. (Eds.) (2012) ¿Qué sabemos de los directores de escuela en Chile? Santiago: CEPPE- Fundación Chile.

Recibido: 10/12/2014

Aceptado: 26/04/2015 